\title{
Evaluating and Planning Flexibility in Sustainable Power Systems
}

\author{
Juan Ma, Student Member, IEEE, Vera Silva, Member, IEEE, Régine Belhomme, Member, IEEE, \\ Daniel S. Kirschen, Fellow, IEEE, and Luis F. Ochoa, Senior Member, IEEE
}

\begin{abstract}
Power systems have traditionally been designed to provide flexibility in a context where demand is met by bulk generation. The integration of variable and uncertain renewable generation sources, such as wind, increases the flexibility needed to maintain the load-generation balance. This paper aims to provide a systematic approach to evaluate the flexibility level and investigate the role of flexibility in generation planning and market operation. An 'offline' index is proposed to estimate the technical ability of both the individual generators and the generation mix to provide the required flexibility. A dedicated unit construction and commitment (UCC) algorithm, able to consider plant investment and operation costs, is developed to determine the optimal investments in flexible generating units. Market simulation models are then implemented to determine the profits obtained from providing flexibility in different market designs. An adapted RTS-96 system is used to test the proposed models and the flexibility metric. Results demonstrate the validity of the UCC algorithm and the coherence of the proposed flexibility index. The analysis of the profits of flexibility shows that the market design plays an important role in the efficient deployment and subsequent profitability of flexibility resources.
\end{abstract}

Index Terms-Flexibility index, wind power, unit commitment and construction, market design, profitability, day-ahead market, balancing market, rolling clearing.

\section{INTRODUCTION}

$\mathrm{L}$ ARGE-SCALE integration of variable renewable energy - poses significant technical challenges mainly due to its variable and hard to predict nature [1-5]. Traditionally, the generation portfolio was designed to provide, in a costeffective and reliable manner, enough flexibility to cope with the variability and the forecast error of demand and with unplanned generation outages. As the generation capacity from variable renewable sources (such as wind power) increases, the system will also need to be able to cope with the variability and uncertainties associated with these sources [68]. The need and cost of providing additional flexibility to integrate large penetrations of intermittent generation has been recognized in $[9,10]$ from a central planning perspective. Nowadays, however, this issue also needs to be addressed from the perspective of the participants in electricity markets. Some of these participants are flexibility providers or potential

J Ma and L F Ochoa are with the School of Electrical and Electronic Engineering at The University of Manchester, Manchester, M13 9PL, UK. (email: juan.ma@postgrad.manchester.ac.uk, luis_ochoa@ieee.org)

V Silva and R Belhomme are with EDF R\&D, Clamart, France. (email: vera.silva@edf.fr, regine.belhomme@edf.fr).

D S Kirschen is with the University of Washington, Seattle, USA. (e-mail: kirschen@washington.edu) providers that will only deliver this flexibility when this returns an economic profit. The electricity market should therefore provide sufficient revenue to make the provision of flexibility profitable in short and long terms.

Previous studies have provided a thorough inventory of the issues related to the requirement of flexibility in wind-power rich system. However, important questions as how to invest in sufficient flexible units to meet these requirements, whether the flexible resources can be rewarded in the market environment and how to evaluate the flexibility level of a system are still waiting for answers. This paper attempts to provide answers to these questions.

Section II of this paper defines the term flexibility in the context of the transition to low-carbon electricity systems and proposes a new index to provide 'offline' evaluations of the flexibility provided by single units and by the system as a whole, to facilitate comparisons, in terms of technical flexibility, between scenarios. Section III describes the UCC algorithm that combines long-term investment and short-term operation costs to determine the optimal construction of flexible generating units. This algorithm is able to define a generation portfolio with sufficient flexibility to cope with high wind power penetrations, while maintaining generation adequacy and system reliability. Section IV presents a model, which considers the day-ahead market, the real-time balancing market and the rolling closure of these markets to evaluate the profitability, for a given generation portfolio, of providing flexibility in a competitive environment. A method to separate the profit stemming from flexibility services from the total profit obtained on the electricity markets is also presented. A case study is presented in section V, with section VI discussing issues surrounding this work. Finally, conclusions are drawn in section VII.

\section{Defining FleXiBle Power Systems}

The term flexibility describes the ability of a power system to cope with variability and uncertainty in both generation and demand, while maintaining a satisfactory level of reliability at a reasonable cost, over different time horizons. In this work, variability and uncertainty from generation are assumed to arise from variable generation, particularly wind power. It is important to highlight that a 'flexible' system, as defined in this work, is a relative concept. For example, given the same demand and available wind generation (WG), it can be said that "system A" is more flexible than "system B" if the former is able to accommodate more WG (i.e., less wind curtailment, 
if at all).

The variations of wind occur on different time scales: seconds, minutes, hours, days, months, seasons and years. In the context of generation scheduling and planning, the objective is to manage the daily net load cycle, the variations of which can be adequately examined on an hourly time scale $[4,11]$. Very fast variations are smoothed out due to the inertia of the large rotating blades of variable speed wind turbines. When considering a large area with geographically dispersed wind farms, the second and minute variations are mitigated by the smoothing effect of wind generation diversity. In addition, variations over longer time scales (days, months, seasons and year) are reflected by the aggregated effect of the hourly variations. Hourly wind data thus covers important information that describes the variability of WG in a large system. To this end, the flexibility is evaluated on an hourly basis in this work. Weekly and seasonal flexibility are considered by running studies covering an entire year.

Two characteristics of wind generation drive the need for flexibility: variability and uncertainty. Frequent and natural fluctuations in wind power pose challenges to conventional generators by creating requirements for fast sudden and large ramping and frequent start-ups [12]. Unavoidable errors between wind power forecasts and actual outputs increase the need for reserves [4, 9, 13, 14]. As a result, to properly accommodate large volumes of wind power, a higher flexibility is required from the system to follow the variable net demand and cope with the uncertainties. These requirements are usually fulfilled by flexible generation, storage, and flexible demand (when available). In this work, given that our focus is on thermal generation flexibility, it is assumed that these requirements are met solely by thermal generation.

Much effort has recently been dedicated to quantify the flexibility of power systems. Most proposed techniques are based on multi-temporal simulation of power system operation [9, 15-17]. Being it clear that a detailed analysis of flexibility requires such a simulation, it is also important to have 'offline' evaluation metrics capable of providing estimations of 'how flexible a system is' and as a result directly comparing the technical flexibility of different systems and generators. To this end, a quantitative metric, which can be used 'offline' to estimate the level of system flexibility and the contribution of individual generators to the aggregated flexibility is highly desirable.

This paper presents a metric to quantify the technical flexibility level of both individual generators and the whole system. This metric quantifies the technical ability of a system or generator to cope with the flexibility requirement resulting from the variability and uncertainty produced by both generation and demand.

Both part-loaded synchronized generators and quick start/shut down generators can be used to meet the flexibility requirement. Part-loaded synchronized (PS) generators can provide upward load following and upward reserves, and their contribution is limited by their ramp-up rate and the spare capacity between their scheduled output and their maximum capacity. Likewise, their ramp-down rate and the difference between their scheduled output and their minimum stable generation (MSG) limit their ability to provide downward load following and downward reserve. Since the constraints for them to provide hourly load following and upward reserve are similar, here we take the constraints for providing reserve as an example.

Mathematically, they can be summarized as follows:

$$
\begin{gathered}
r_{\text {up }}(i, t) \leq \min \left\{P_{\max }(i)-P g(i, t), \operatorname{Ramp}_{\text {up }}(i) \Delta t\right\} \\
r_{\text {down }}(i, t) \leq \min \left\{P g(i, t)-P_{\text {min }}(i), \operatorname{Ramp}_{\text {down }}(i) \Delta t\right\} \\
\forall i \in P S, \forall t \in T
\end{gathered}
$$

Here, $r_{u p}(i, t)$ and $r_{\text {down }}(i, t)$ represent the up and downward reserves that can be provided by conventional generator $i$ at hour t. $P_{\max }(i)$ and $P_{\min }(i)$ are the maximum capacity and the MSG of conventional generator i. $\operatorname{Ramp}_{\text {up }}(i)$ and $\operatorname{Ramp}_{\text {down }}(i)$ are respectively the ramping up and down rates of generator $i$ per hour. $\Delta t$ corresponds to one hour. $P_{g}(i, t)$ is the scheduled output of generator $i$ at hour $t$.

Generating units who can quick start/shut down (QS) within the required time scale, e.g. less than one hour, can also provide up/down reserve and upward/downward load following. Their contribution to reserve is formulated as:

$$
\begin{aligned}
& r_{\text {up }}(i, t) \leq \min \left\{P_{\max }(i), \operatorname{Ramp}_{\text {up }}(i) \Delta t\right\} \\
& r_{\text {down }}(i, t) \leq \min \left\{P g(i, t), \operatorname{Ramp}_{\text {down }}(i) \Delta t\right\} \quad \forall i \in Q S, \forall t \in T
\end{aligned}
$$

The contribution of each generating unit to flexibility is thus constrained by its deployable range. Hence, the ability of the system to follow the variability of net demand and meet the demand balance constraint will be constrained by ramping capabilities and adjustable capacity.

From (1) and (2) it is possible to see that the ramp rates and the difference between the MSG and the capacity of a plant are the parameters that define its capability to provide flexibility. Based on this, a flexibility index can be defined for each conventional generator $i$. To allow comparisons, this index needs to be normalized as follows to account for the sizes of the units:

$$
f l e x(i)=\frac{1 / 2\left[P_{\max }(i)-P_{\min }(i)\right]+1 / 2[\operatorname{Ramp}(i) \cdot \Delta t]}{P_{\max }(i)}, \forall i \in A
$$

where $1 / 2 \operatorname{Ramp}(i)$ is the average value of $\operatorname{Ramp}_{\text {up }}(i)$ and $\operatorname{Ramp}_{\text {down }}(i)$, and thus indicates the speed at which a unit can adjust its output within $P_{\max }(i)-P_{\min }(i)$. Notice that for QS units, the $P_{\min }(i)$ is replaced by 0 . Here the $\operatorname{Ramp}_{\text {down }}(i)$ refers only to normal operating strategies. Outages are not considered when investigating flexibility resources.

The flexibility index of a whole system $\mathrm{A}$ is then defined as the weighted sum of the flexibility indices flex( $i)$ of the individual generators. The weighting factors are taken as equal to the capacity contribution of each unit. The whole system flexibility is thus calculated as follows: 


$$
F L E X_{A}=\sum_{i \in A}\left[\frac{P_{\max }(i)}{\sum_{i \in A} P_{\max }(i)} \times \operatorname{flex}(i)\right], \forall i \in A
$$

Although power system operation can be very complex and variable, this index is not affected by operational decisions. It thus provides a simple method to assess the technical ability of power systems to accommodate renewable energy sources.

\section{Designing A FleXible Generation PortFolio}

This section presents a method to determine the set of generating units that provides optimally the flexibility required to accommodate a given wind penetration. Such an optimization must bridge the gap between the long-term investment decisions about the plants to be built and the shortterm operational decisions of how these plants are scheduled. This is achieved in this work by introducing a new optimization algorithm, called the Unit Construction and Commitment (UCC). This algorithm is based on the unit commitment (UC) algorithm which enforces the flexibility constraints, such as ramping rate, minimum stable generation and minimum up/down time. This optimization thus determines whether a plant should be built to provide additional flexibility at a reasonable cost. Significant modifications are required to transform a traditional UC algorithm into an algorithm capable of balancing the long- and short-term costs of providing flexibility:

- The optimization algorithm must be able to decide not only when a particular generating unit should be started and shut down, but also whether building that unit is optimal or not.

- The objective function must include not only the operating cost but also the amortized investment cost of each generating unit.

- The optimization horizon must be sufficiently long in order to capture the intra-day, daily and seasonal variations in load and wind generation that drive the need for flexibility.

- It should explicitly specify the wind integration target. Therefore, the optimization problem considers not only generation adequacy but also flexibility adequacy (i.e. the flexibility level needed to attain a specific wind integration target)

The main extensions from the classic UC algorithm to the proposed UCC are summarized in Table I.

In a conventional UC problem, all the generators are scheduled under the premise that they already exist in the system. In other words, the generation mix considered in a conventional UC problem is a fixed set of available generating units. Instead, the proposed UCC should have the opportunity to add generating units from an available set. The existence (or non-existence) of those new generating units will be modelled using additional binary decision variables.

\section{A. Unit Construction and Commitment}

Unlike a traditional UC where the objective function minimizes only the operational costs of existing units, the
TABLE I MAIN EXTENSIONS FROM UC TO UCC

\begin{tabular}{|c|c|c|}
\hline & UC & UCC \\
\hline Decision variables & $\begin{array}{c}\text { On/off status of } \\
\text { generators (binary) } \\
\text { Output of generators } \\
\text { (continuous) }\end{array}$ & $\begin{array}{c}\text { On/off status of } \\
\text { generators (binary) } \\
\text { Output of generators } \\
\text { 'Existence' of } \\
\text { generators (binary) }\end{array}$ \\
\hline Objective function & Operational cost & $\begin{array}{l}\text { Operational cost } \\
\text { Investment cost }\end{array}$ \\
\hline Optimization horizon & $24 \mathrm{~h}-168 \mathrm{~h}$ & One year \\
\hline Constraints & $\begin{array}{l}\text { System-level } \\
\text { constraints } \\
\text { Generator-level } \\
\text { constraints }\end{array}$ & $\begin{array}{c}\text { System-level constraints } \\
\text { Generator-level } \\
\text { constraints } \\
\text { Wind curtailment } \\
\text { constraint }\end{array}$ \\
\hline
\end{tabular}

UCC algorithm minimizes simultaneously both the operational cost of all the existing and new units and the investment costs of new units. Equation (5) shows the objective function of this algorithm.

$$
\min \left(\sum_{i=1}^{N} \sum_{t=1}^{T} O C(i, t)+e_{j}\left(\sum_{j=1}^{A} \sum_{t=1}^{T} A O C(j, t)+\sum_{j=1}^{A} A I C(j)\right)\right)
$$

Here $O C(i, t)$ is the operational cost of existing unit $\underline{i}$ at time $t$; $A O C(j, t)$ is the operating cost of the additional unit $j$ at time $t$; and $A I C(j)$ is the investment cost of unit $j$ amortized over the optimization horizon. $e_{j}$ is a binary decision variable which indicates whether the additional flexible unit $j$ should be built. A value of ' 1 ' for this decision variable indicates that the corresponding generating unit exists and can be committed. A value of ' 0 ' indicates otherwise.

Equation (6) defines the amortized investment cost over one year:

$$
\operatorname{AIC}(j)=C_{M W}(j) P_{M A X}(j)\left(\sum_{n=1}^{L(j)} \frac{1}{(1+R O I)^{n}}\right)^{-1}
$$

(6)

where $C_{M W}(j)$ is the cost per MW of building unit $j . P_{\max }(j)$ is the capacity of unit $j . L(j)$ is the expected lifetime of unit $j$. $R O I$ the is return on investment. The larger the $R O I$ is, the larger the amortized investment cost is, which implies a larger proportion of investment cost in the total cost (objective function). The investment cost of a generating unit is taken into consideration only if this unit has been built $\left(e_{j}=1\right)$ and is thus available for commitment. The operational cost $O C(i, t)$ considers incremental cost and start-up cost, as shown in $(7)$.

$O C(i, t)=u(i, t)(\operatorname{INC}(i) \times p(i, t))+u(i, t)(1-u(i, t-1)) \operatorname{SC}(i)$

where $u(i, t)$ is the decision variable indicating the on/off status of generator $i$ at hour $t . p(i, t)$ is the continuous decision variable showing the output of generator $i$ at hour $t$. INC(i) and SC(i) represent the incremental and start-up costs of generator $i$, respectively.

The objective function is subject to the traditional UC 
constraints: power balance constraint, spinning reserve, maximum and minimum generation limits, minimum up/down time and ramp rate limits, as well as a new wind curtailment constraint.

\section{1) Power balance}

The total power generated must be equal to the demand in all time intervals.

$$
\sum_{i=1}^{N} p(i, t) u(i, t)+\left[W_{f}(t)-w_{c}(t)\right]=D_{f}(t) \quad \forall t=1, \ldots, T
$$

where $W_{f}(t)$ is the forecasted wind power generation at hour t. $w_{c}(t)$ is the decision variable representing the curtailed wind generation at hour $t . D_{f}(t)$ is the forecasted demand at hour $t$.

\section{2) Spinning reserve}

In this work, the uncertainty from the wind forecast error is considered through the spinning reserve requirement. Here, the wind forecast errors $\varepsilon(t)$ are modelled as zero-mean normally distributed random variables. At each time period, the wind forecast error is fitted to a normal distribution with standard deviation $\sigma_{w}(t)$. Statistical models for wind speeds at specific locations do not fit normal distributions and the non-linear relation between wind speed and power output further distorts the statistical distribution. However, wind generation is assumed here to be aggregated from a large number of widely dispersed wind turbines. This justifies the use of the central limit theorem and the assumption that the forecast error is normally distributed.

The spinning reserve requirement $r(t)$ must be increased to be able to cope with not only the sudden loss of the largest generating unit but also a simultaneous error in the wind power. In addition, this reserve has to be sufficient to cover most of the wind forecast error; hence an additional reserve equal to 3.5 times the standard deviation of the wind forecast error is adopted [8].

$$
r(t) \geq \max \left(u(i, t) \times P_{\max }(i)\right)+3.5 \sigma_{w}(t)
$$

3) Minimum and maximum generation limits

Generating units must be operated within their minimum stable generation and maximum capacity.

$$
P_{\min }(i) \leq p(i, t) \leq P_{\max }(i)
$$

4) Minimum up/down time and ramp rates

The operation of the generating units is also subject to a number of technical constraints such as minimum up- and down-times, ramp-up and -down limits and generation limits among others, [18-19]. These are enforced by sets of inequality constraints which, for the sake of simplicity here are denoted as follows:

$$
\mathrm{h}(\mathbf{u}, \mathbf{p}) \leq 0
$$

\section{5) Wind curtailment}

While a conventional generation portfolio could be designed to accommodate any amount of WG it may not always be economically justified to do so. In some cases, the flexibility could be best provided by the wind generators themselves under the form of wind curtailment. Given that this is not desirable the tolerable amount of wind curtailment can

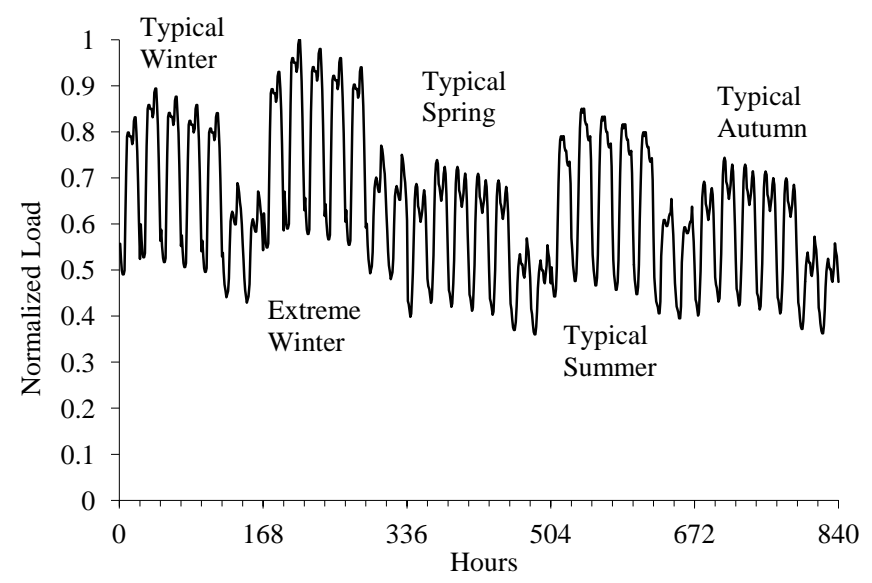

Fig. 1. Load profile with four seasonal representative weeks and one extreme winter week

be treated as an exogenous design variable and introduced into the UCC problem using an additional constraint on the extent of wind curtailment over the optimization horizon:

$$
\sum_{t=1}^{T} w_{c}(t) \leq K_{c} \times \sum_{t=1}^{T} w g(t)
$$

where $w g(t)$ and $w_{c}(t)$ are respectively the wind power production at period $t$ and the wind power curtailed at period $t$. $K_{c}$ is the proportion of wind power that can be curtailed over a year. This constraint ensures that enough additional flexibility will be built to accommodate at least $1-K_{c}$ times the available WG over a whole year.

\section{B. Representative Weeks}

When considering the needs for flexibility, one should take into account not only the hourly but also weekly and seasonal variations in demand profile and WG that may occur during a year. However, running the proposed optimization algorithm over a whole year with an hourly resolution requires an excessive amount of computing time. To reduce the last to an acceptable level, a set of representative weeks are used to simulate a whole year. By selecting the most challenging operational weeks (e.g., peak demand with no wind, extremely variable wind power generation, etc.) throughout the year, it is possible to understand the 'worst case scenario' flexibility requirements of a given wind penetration level. However, in order to capture the economic aspects of integrating large volumes of wind and bringing new players to the provision of flexibility, the annual electrical energy generation should be calculated.

In this work, each season is taken into account using a representative week. Furthermore, to ensure the generation adequacy under extreme situations, a week with extremely high demand and no wind is also considered. Fig. 1 illustrates such a load profile with the extreme week inserted between the typical winter and spring weeks. Load profiles of the four typical weeks are obtained using historical 'average' values of load during each season. Since the load profile usually follows typical weekly and daily patterns, the average weekly values 
represent the general variations of the demand in the corresponding season. In addition, it guarantees that the typical week has the same load factor as the season it represents.

For the case of WG it is in general not possible to find such 'representative' weeks since there are no repetitive daily or weekly patterns. As a consequence, average values are not sufficient to model the wind profile. In order to capture the 'representative' wind variability, two criteria were considered in this work:

- Wind profiles have to keep the same wind capacity factor as the corresponding seasons. This is to guarantee that the original wind-energy penetration level is not altered.

- Variations in wind generation must display the worstcase scenario for the corresponding season. In this way, the flexibility requirement due to the variability of wind will not be underestimated

For instance, for the winter months, the hourly WG output of week $k$ in winter $(W)$ is firstly scaled up or down according to the average wind capacity factor for the whole season. A scale factor $(S F)$ is obtained by dividing the wind capacity factor for the season by that of each corresponding week. Thus, for each winter week $(k)$, the hourly WG is modified as follows:

$$
w(k, t)=w_{\text {original }}(k, t) \times \frac{C F_{\text {season }}}{C F_{\text {week }}(k)} \quad \forall t \in T, k \in W
$$

where $C F_{\text {week }}(k)$ represents the weekly wind capacity factor, $C F_{\text {season }}$ is the winter wind capacity factor, $w_{\text {original }}(k, t)$ is the original WG output at hour $t$ in week $k$, and $w(k, t)$ is the modified WG output at time $t$ in week $k$.

Then, the aggregated hourly variations in each week are calculated. This is done by adding up the absolute values of hourly variations (independent of being upward or downward variations). Finally, the week with the largest aggregated variations is selected as the representative week for winter. The selected week maintains the same capacity factor as the winter season and at the same time represents the worst-case scenario of variations in wind profile. Representative wind profiles for the other three seasons, spring, summer and autumn, are constructed using the same method.

Because only five representative weeks are used, the objective function must reflect how many weeks each of them represents. Table II shows the weights that are applied to the weekly operating costs if we assume that an extreme winter week happens every four years.

These representative week profiles cannot be directly connected in the algorithm because of the significant influence that the initial state of the units (on/off status, time since last status change, dispatched output) can have on the scheduling decisions. An incorrect initialization could bias the calculation on flexibility requirements.

Since each of these weeks represents an 'average' of all the weeks of a particular season, one can make an assumption that this is followed by another similar week. It is thus reasonable to assume, as illustrated in Fig. 2, that the final state of each representative week is identical to its initial state [20].
TABLE II

TYPICAL WEIGHTING FACTORS FOR REPRESENTATIVE WEEKS

\begin{tabular}{c|c}
\hline Week & Weighting \\
\hline Average winter & 16.75 \\
\hline Extreme winter & 0.25 \\
\hline Average spring & 9 \\
\hline Average summer & 13 \\
\hline Average autumn & 13 \\
\hline
\end{tabular}

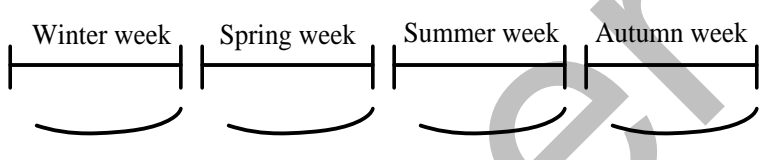

Fig. 2. Initialization of representative weeks

\section{Assessing the Profitability Of FleXibiLity}

The tool presented in section III can be used by policy makers and system operators to determine the generation portfolio required to achieve the flexibility required to meet a given WG target. In a market-based system, however, it is the profitability of the generators that will drive investments. This section describes a method to quantify this profitability using a market model that takes into account both day-ahead and realtime balancing markets. This tool can be used to assess the impact of the market design on the profitability of providing flexibility.

\section{A. Day-ahead market}

This model assumes a perfectly competitive market where decisions are strongly affected by physical constraints. This eliminates the effects of market power and reveals the true value of flexibility. In a perfectly competitive market every participant bids its true operating cost and all bidders are paid at the hourly uniform market clearing price. Such a market can be modeled using centralized UC scheduling. The conventional generators that are scheduled to hold back their power outputs to provide reserve receive a uniform option fee for each MWh provided (e.g. 5\$/MWh in this work). This option fee gives to the system operator the right to buy this reserve at the exercise price in the real-time balancing market [21]. Generators are thus able to profit from selling both energy and reserve in the day-ahead market.

In the day-ahead market, the wind forecast is simulated based on the realization of WG and the simulation of wind forecast error. The realization of WG is assumed to be the same as historical data. Wind forecast error is simulated based on a probability distribution considering the associated temporal correlation [22, 23]. Demand forecast is also simulated using this approach.

\section{B. Real-time balancing market}

The real-time balancing market clears the deviations of the generation schedule caused by the aggregated wind and demand forecast error. The real-time balancing market is also assumed to be perfectly competitive, i.e. the bids for up and down adjustments are equal to the actual marginal production costs. By selling flexibility at marginal cost, the generating 
units provide upward reserve for compensating the unforecasted of WG or an under-forecast of demand. On the other hand, when a surplus of WG or less than anticipated demand happens, the generating units provide down regulation by reducing the generation output scheduled in the day-ahead market. The real-time balancing market is assumed to be cleared on an hourly basis and the clearing price is determined by the bid for the last MWh of reserve required. Although generators are likely to bid their up reserve higher than marginal cost (because of the time value of reserve), we neglect this phenomenon in our model to be able to calculate the actual value of flexibility.

In the real-time balancing market, generators can thus make a profit by selling their up-reserve or by holding back its output to provide downward reserve.

\section{Rolling clearing of the day-ahead market}

Given that wind forecast information is usually updated several times a day, a rolling clearing mechanism can facilitate the efficiently deployment of flexibility resources. The use of rolling clearing in systems with WG was originally proposed in [24], and has also been applied in [25]. The underlying principle of rolling-clearing is that instead of carrying out the commitment once a day the commitment is carried out more frequently. For example, in a 6-hour rolling commitment the first commitment is performed with a 24-hour horizon, then the system is 'rolled' forward 6 hours and the stochastic parameters such as wind and load forecasts are updated. The system operator then re-schedules the opening positions. The day-ahead and real-time balancing markets are thus re-cleared with the latest information. For example, if the wind forecast is updated every 6 hours, the day-ahead market will be cleared 4 times per day, using the latest wind forecast.

Fig. 3 shows the resulting market structure with day-ahead and real time balancing market.

Likewise, for the real time balancing market, the system operator calculates the deviations between the actual WG and the latest wind forecast information. Then it procures flexibility to handle positive or negative deviations. Therefore, the closer the wind forecast gets to the real time, the more accurately the required flexibility is traded.

The balancing market is cleared in real time according to the differences between the actual WG and the latest wind forecast error with a 6-hour lead-time.

The use of rolling markets reduces the uncertainty that the system operator needs to cope with because it relies on shorter forecast horizons and therefore reduces the requirement for flexibility. The cost of providing flexibility to the system as a whole is thus reduced and the economic efficiency of the system is improved.

This, however, reduces to a certain extent the profit of those units providing flexibility and reduces the incentive for investments in flexibility.

\section{CASE STUDY}

In this section, the three approaches previously proposed, i.e., the flexibility index, the UCC, and the market-based flexibility profit evaluation, are applied to the IEEE Reliability

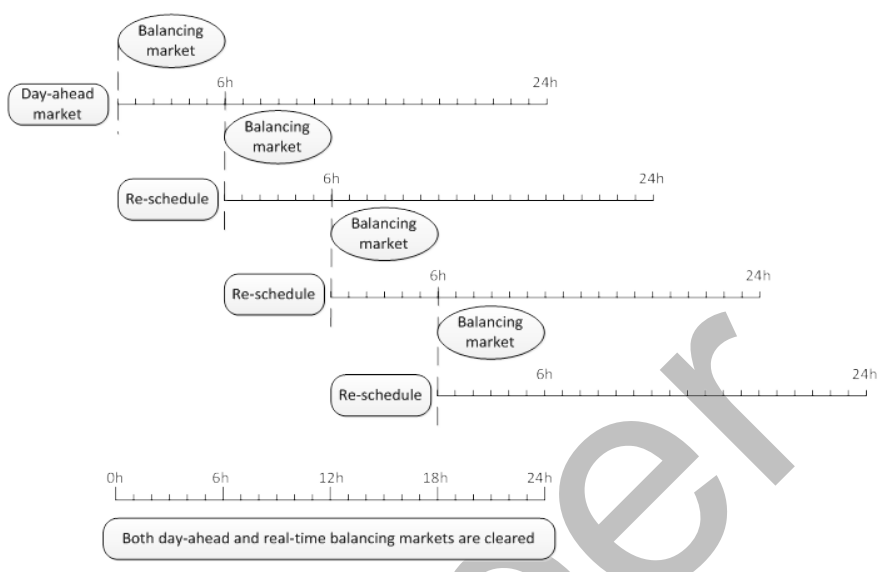

Fig. 3. Re-clearing of day-ahead and balancing markets with updated wind forecast every 6 hours.

TABLE III

FLEXIBILITY INDEX FOR THE 26-UNIT RTS SYSTEM

\begin{tabular}{c|c}
\hline Name & Flexibility Index \\
\hline 26-unit system & 0.5352 \\
\hline U12_Oil/Steam & 0.8000 \\
\hline U20_Oil/CT & 0.2100 \\
\hline U76_Coal/Steam & 0.7266 \\
\hline U100_Oil/Steam & 0.6875 \\
\hline U155_Coal/Steam & 0.5395 \\
\hline U197_Oil/Steam & 0.5204 \\
\hline U350_Coal/Steam & 0.4357 \\
\hline U400_Nuclear & 0.4691
\end{tabular}

TABLE IV

WIND UTILIZATION RESULTS OF DEMONSTRATION SYSTEMS WITH DIFFERENT

\begin{tabular}{|c|c|c|c|}
\hline \multicolumn{4}{|c|}{ FLEXIBILITY INDEX } \\
\hline $\begin{array}{c}\text { Generation Mix } \\
\text { APL }=2200 \mathrm{MW} \\
\text { Wind Capacity }=880 \mathrm{MW}\end{array}$ & $\begin{array}{l}\text { Total } \\
\text { capacity } \\
\text { (MW) }\end{array}$ & $\begin{array}{l}\text { Flexibility } \\
\text { Index }\end{array}$ & $\begin{array}{c}\text { Wind } \\
\text { Energy } \\
\text { Utilization }\end{array}$ \\
\hline HFM $\quad 9 *(\mathrm{U} 12+\mathrm{U} 76+\mathrm{U} 100+\mathrm{U} 155)$ & 3087 & 0.6333 & $80.59 \%$ \\
\hline $\begin{array}{ll}\text { MFM } & 7 *(\mathrm{U} 12+\mathrm{U} 76+\mathrm{U} 350+\mathrm{U} 20) \\
\end{array}$ & 3206 & 0.4836 & $41.99 \%$ \\
\hline LFM $\quad 4 *(\mathrm{U} 197+\mathrm{U} 400+\mathrm{U} 350+\mathrm{U} 20)$ & 3868 & 0.4621 & $10.67 \%$ \\
\hline 26units system (Base_Mix) & 3105 & 0.5352 & $48.48 \%$ \\
\hline
\end{tabular}

Test System (RTS-96) [26] (without hydro generation). The UCC and the market-based profit evaluation have been formulated as mixed integer linear programming (MILP) problems and implemented using the Dash Xpress optimization solver [27]. The physical parameters of the units were obtained from [26] and [28]. Using a PC with Intel Quad Core Processor and 6GB of RAM, the average computation time for the UCC was 10 hours, whereas for the rolling clearing of the day-ahead market for the simulation of one year it was about 30 minutes.

\section{A. Flexibility index}

Table III shows the flexibility indices calculated for representative generating units and for the whole test system using the method proposed in section II. By comparing the flexibility index of a particular unit with the index for the whole system, we can assess how much it contributes to the flexibility of the overall system. If its index is higher than the system's flexibility index, this unit can be considered as flexible in this system. Vice versa, inflexible units are those 
with a flexibility index lower than the system level. For example, in this case, the 26-unit system (designated as base_mix) has a flexibility index of 0.5352 . Units U12, U76, $\mathrm{U} 100$, and U155 can therefore be considered as the flexible units, while U20, U197, U350 and U400 are inflexible or low flexibility units.

This flexibility index is then used to create sets of units with comparable total capacities but different levels of flexibility. These sets are combined to create the generation mixes with different levels of flexibility index that are used in our studies. For example, a highly flexible mix (HFM) is composed by nine replications of units U12, U76, U100, and U155. The same principle is used to create the medium (MFM) and low flexibility mixes (LFM). Table IV shows the composition of each mix in terms of units, total installed capacity, and aggregated flexibility index.

The performance of these three sets of generating units, in terms of their ability of accommodating WG, was tested on a scenario where they had to meet the same annual load profile with the same WG profile, using a standard unit commitment algorithm. The normalized demand and wind profiles were separately multiplied by the annual peak load of 2200MW and wind capacity of $880 \mathrm{MW}$, respectively. The simulation results obtained, in terms of total wind energy used to supply demand designated as wind energy utilization, are also presented in Table IV.

As expected, the High Flexibility Mix (index 0.6333) is able to utilize $81 \%$ of the wind energy available which is a considerably larger fraction than the other two groups. The Low Flexibility Mix (index 0.4621) is only able to accommodate a little over $10 \%$ of the available wind energy, which means that about $90 \%$ of the WG has to be curtailed due to generation flexibility constraints.

Table IV also shows that the Base_Mix of 26 units has a flexibility index of 0.5352 , which is between the values for the high and medium flexibility mixes. This is supported by its wind energy usage (48.48\%) that is also in between those of the two groups (80.59\% and $41.99 \%$ ).

\section{B. Planning a flexible generation portfolio}

The ability of the UCC algorithm to determine the optimally flexible generation portfolio was also tested using the Base_Mix (26 units). This system originally serves a load profile with an annual peak load (APL) of 2500MW and a load factor of 0.65 . The wind power output, for the whole year, is represented by an hourly time series, obtained from normalized historical data [29]. This has a capacity factor of 0.22 . The simulated WG output, for different scenarios, is calculated by multiplying the normalized hourly time series by the installed wind capacity.

The maximum annual wind curtailment is assumed to be $20 \%\left(K_{c}\right)$ of the total available WG. This (rather high) value is used due to the limited flexibility originally present in the RTS-96 test system but serves the purpose of demonstrating the proposed methodology. In practice, the curtailment level should be determined on economic grounds. To perform the simulation the installed wind capacity (represented in \% of
APL = Installed Wind Capacity/Annual Peak Demand) is used

TABLE V

\begin{tabular}{c|c|c|c|c|c|c}
\hline & $\begin{array}{c}\text { Pmin } \\
\mathbf{( M W )}\end{array}$ & $\begin{array}{c}\text { Pmax } \\
\mathbf{( M W )}\end{array}$ & $\begin{array}{c}\text { Ramp } \\
\text { rate } \\
\mathbf{( M W / h )}\end{array}$ & $\begin{array}{c}\text { Investment } \\
\mathbf{C o s t} \\
\mathbf{( k \$ \mathbf { M W } )}\end{array}$ & $\begin{array}{c}\text { Fuel Cost } \\
\mathbf{( \$ / M W h )}\end{array}$ & $\begin{array}{c}\text { Flexibility } \\
\text { Index }\end{array}$ \\
\hline $\begin{array}{c}\text { Low } \\
\text { Flexible }\end{array}$ & 100 & 200 & 70 & 2117 & 18 & 0.4250 \\
\hline $\begin{array}{c}\text { Medium } \\
\text { Flexible }\end{array}$ & 50 & 200 & 100 & 536 & 250 & 0.6250 \\
\hline $\begin{array}{c}\text { High } \\
\text { Flexible }\end{array}$ & 10 & 200 & 150 & 409 & 3700 & 0.8500 \\
\hline
\end{tabular}

TABLE VI

INVESTMENT DECISION UNDER DIFFERENT TARGETS OF WIND CAPACITY

\begin{tabular}{|c|c|c|c|c|c|c|}
\hline $\begin{array}{c}\text { Base case } \\
\text { Max } \\
\text { wind } \\
\text { 1100MW }\end{array}$ & $\begin{array}{c}\text { New } \\
\text { Target } \\
\text { for wind } \\
\text { (\% APL) }\end{array}$ & $\begin{array}{c}\text { Type } \\
\text { for unit }\end{array}$ & $\begin{array}{l}\text { Low } \\
\text { Flex }\end{array}$ & $\begin{array}{l}\text { Medium } \\
\text { Flex }\end{array}$ & $\begin{array}{c}\text { High } \\
\text { Flex }\end{array}$ & $\begin{array}{c}\text { System } \\
\text { Flex } \\
\text { Index }\end{array}$ \\
\hline Case 1 & 46 & & priou & & & 0.5285 \\
\hline Case 2 & 48 & $\gtreqless:$ & & Option & & 0.5406 \\
\hline Case 3 & 50 & & & & Option & 0.5543 \\
\hline
\end{tabular}

as an input parameter. To find the maximum capacity that the system can accommodate without exceeding 20\% wind curtailment, this parameter is increased until the UCC becomes infeasible. In this system, the maximum feasible installed wind capacity was found to be $1100 \mathrm{MW}$ or $44 \%$ of APL. Given that the annual demand is $14.2 \mathrm{TWh}$, the annual available WG is $1100 \times 8760 \times 0.22=2.1 T W h$. The annual utilized WG is taken to be $20 \% \times 2.1 T W h=1.68 T W h$. Thus, the maximum percentage of total annual demand served by WG is $1.68 / 14.2=11.83 \%$.

Whenever the maximum allowable wind capacity is reached (1100MW), it means that the maximum wind energy that this system can accommodate is obtained (1.68TWh). It implies that any extra wind capacity installed will result in a wind utilization factor lower than $80 \%$. Therefore, in order to further increase the wind capacity and maintain a high wind utilization factor, new investments in flexibility are needed.

In order to analyze how to investment in flexibility, let us assume three candidate generating units, of the same capacity and different flexibility indices and costs, are being considered as possible investment alternatives but only one of these will be built. Table $\mathrm{V}$ gives the key parameters for these three units. Large differences in fuel costs have been chosen to investigate whether a very flexible unit has to be built to handle high wind penetration even if its operating cost is extremely high (3700\$/MWh).

Table VI shows that when the total wind capacity is increased from 1100MW to 1150MW (Case 1), the optimization chooses the low flexibility unit because it has the lowest operating cost. If the target wind capacity is increased to $1200 \mathrm{MW}$ (Case 2), the medium flexibility unit is selected because the low flexibility unit does not sufficiently enhance the system flexibility to meet the constraints. When the target is 1250MW (Case 3), the highly flexible unit is selected even though it has an extremely high operating cost. The last 
column of Table $\mathrm{V}$ lists the flexibility indices of the three new portfolios (26 units +1 new invested unit) and shows that there is a good correlation between the proposed flexibility index and the amount of wind capacity that can be integrated.

\section{Profitability of flexibility}

Generally speaking, flexibility is profitable in the dayahead market for two main reasons:

- More flexible units are required to meet the constraints that are exacerbated by the need to integrate additional WG into the generation mix. This means that more flexible peaking units become frequently the marginal units. This increases the market-clearing price in some of the periods, and each MWh of energy sold generates higher revenue. This additional revenue benefits all scheduled units and not just the flexible ones; and,

- The increased reserve requirement produces increased revenues from option fees for the units that have the technical ability to provide reserve. When this reserve is deployed in the real-time balancing market, further revenues from the exercise fees are obtained by these units.

However, increasing the penetration of WG, to supply the same level of demand, decreases the output of conventional generators. As a result, the overall profit of conventional generating units is reduced. Nevertheless, given that WG is variable (and long periods of low wind can be observed) and uncertain (the needs for reserve increase), these generators are

still required to ensure system reliability and adequacy. An appropriate market design is therefore required, so that these units are rewarded for their contribution to the system.

Moreover, given the need for flexibility to accommodate WG the right economic signals need to be sent to encourage the investment in flexible generation. As a consequence, the true value of flexibility cannot be measured and rewarded based on the absolute value of the profits of conventional generators. In order to extract this value from the results of our studies, the profits obtained from the different markets (energy and reserve) are normalized on the basis of the energy and reserve capacity traded by all generating units. Formally, the overall normalized profit of a generation mix is calculated by dividing the sum of its profits from the day-ahead and balancing markets by the algebraic sum of the energy it traded in these markets:

$$
\bar{\Omega}=\frac{\Omega_{D A}+\Omega_{R T}}{\sum P g+\sum \operatorname{Re}_{u p}-\sum \operatorname{Re}_{\text {down }}}
$$

where $\bar{\Omega}$ is the normalized profit; $\Omega_{D A}$ is the total profit obtained from day-ahead market; $\Omega_{R T}$ is the total profit obtained from balancing market; $\Sigma P g$ is the total power traded in day-ahead market; $\Sigma \mathrm{Re}_{\text {up }}$ is the total upward reserve deployed; and, $\Sigma \mathrm{Re}_{\text {down }}$ is the total downward reserve deployed.

Fig. 4 shows the overall normalized profit for the Base_Mix (26 units). The demand (with APL at 2500 MW) and wind profiles are the same as used in the previous section.

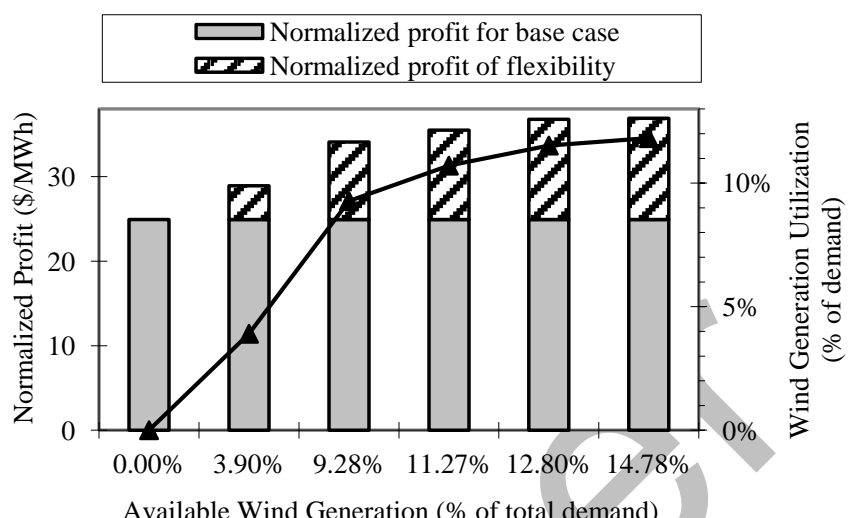

Fig. 4. Normalized profit and wind energy utilization under different available WG penetration

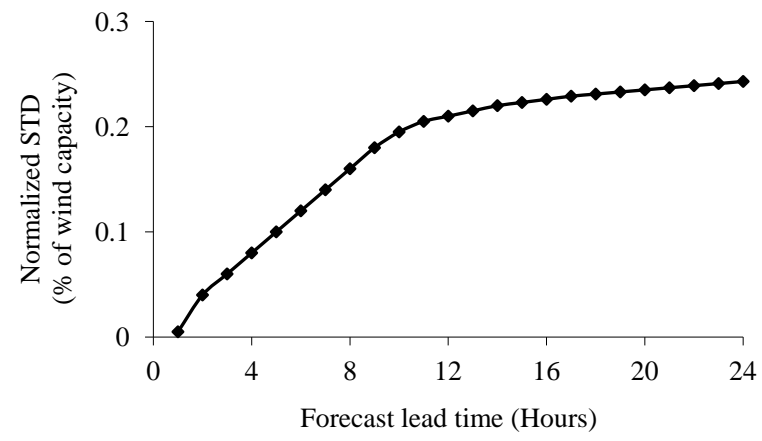

Fig. 5. Performance of wind forecast errors (normalized standard deviation) dependency on forecast length.

The horizontal axis shows the available wind energy in \% of the annual energy demand. The normalized profit is shown on the left vertical axis. The right vertical axis indicates the percentage of total energy demand that is actually served by WG. The shaded areas show the additional normalized profit compared to the case without WG. In essence this shows the total value of flexibility required to accommodate WG. The results show that this value increases with the wind penetration, but saturates as the wind capacity approaches its limit. As shown in the previous section, under the constraint of $K_{c}=20 \%$ (max wind energy curtailed), the Base_Mix system nearly approaches its upper limit for accommodating wind energy above $11.85 \%$ of total annual demand. These conditions are observed when the WG available represents $14.78 \%$ of the annual energy demand. Above this, the profit that conventional generators obtain from flexibility saturates since any additional WG will be curtailed.

This corresponds to having WG providing the remaining flexibility required to meet the system operation constraints. Wind curtailment, however, should be limited to ensure a good environmental performance of the system, therefore, alternative means of flexibility need to be found so that additional wind capacity can be added to the system.

\section{Effect of rolling commitment}

As shown in Fig. 5 [30], the wind forecast error increases with the forecast lead-time. When this horizon is reduced, the 
uncertainty decreases, and less additional reserve is required to

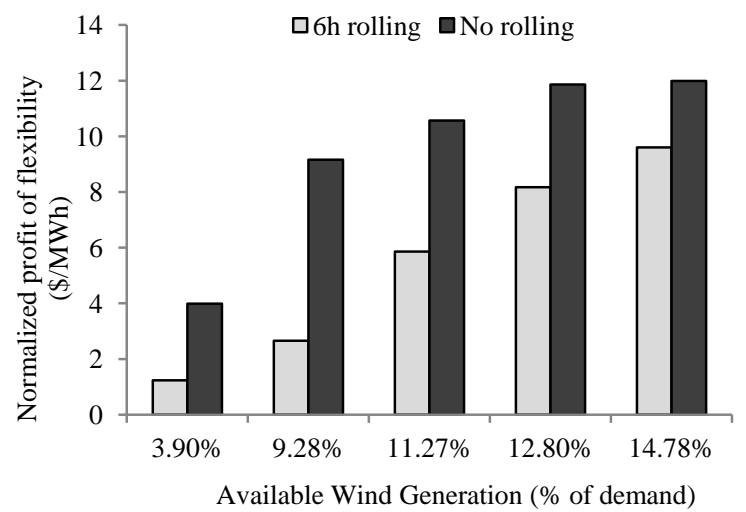

Fig. 6. Effects of rolling clearing day-ahead market on the value of flexibility

cater for wind uncertainty. This has an effect on the economic performance of the system, given that reserve represents an important share of the system operating cost. In this work, the reserve market is cleared at the same time as the energy market through co-optimization.

For a market design where energy and reserve markets are cleared on a day-ahead basis, the system operator needs to procure reserve to cover the 24-hour wind forecast uncertainty. Rolling market clearing takes advantage of better wind forecasts. This affects the overall system cost and the profitability of generating units that provide flexibility. The difference between the two market designs is shown in Fig. 6, in terms of normalized profits achieved with a forecasting and scheduling horizon of 24 hours, and a rolling 6-hour market clearing. In both cases the Base_Mix is used. These results show that using rolling clearing reduces the overall profitability of flexibility. This shows that, by changing the market design, for the same generation mix the cost of accessing flexibility to accommodate WG is reduced. This result indicates that the cost of the flexibility is driven not only by the technical flexibility of the generation mix but also by the design of the markets where this flexibility is traded. A more efficient market design is one that reduces the exposure to wind uncertainty, which reduces the physical flexibility requirement, and, consequently, the cost of physical flexibility. Therefore, an efficient market design can be a nontechnical source of flexibility.

Implementing a rolling market clearing is not a simple matter but it represents an idea that is worth pursuing as an alternative to investing in new flexible plants that may have a low utilization factor.

\section{DISCUSSION}

It is important to highlight that the relationship between the flexibility of a system and the operational state of each unit will depend on how flexibility is defined. If flexibility is defined as time-specific, e.g., the flexibility that a system can provide in each time interval, then it would closely be linked to the operational level of each unit at that specific time (and the flexibility level is different for each time interval). In this work, flexibility is defined as the aggregated ability of a system to cope with uncertainties and variations. In terms of the proposed flexibility indices, the corresponding flexibility can be considered -to some extent- to be non-time specific.

The methodology proposed here uses a deterministic approach to address wind uncertainty, which is inherently stochastic. Further development should consider more sophisticated approaches such as stochastic programming.

In order to simplify the validation of the proposed flexibility analyses, thermal generators were used throughout this work as the only means of providing flexibility. The proposed approach can be extended to include interconnections, storage and demand side response. For example, the unit construction and commitment algorithm can be adapted to determine the optimal addition of other flexibility resources [31]. Alternative sources of flexibility and the inherent complexity of the problem have been investigated in [9].

An hourly resolution for the unit commitment cannot indicate the flexibility requirement within the hour. This work aims to provide a planning model for a large geographically dispersed system. Therefore, the intra-hour variations of wind and demand are not taken into consideration. However, the proposed model could be extended to include very short-term flexibility in order to increase its accuracy.

The four representative weeks are able to capture the natural variations in demand and wind while maintaining the original load and wind capacity factors, respectively. Provided there are no significant external factors that affect the wind resource and/or the demand, it is likely that for a given year these representative weeks will be very similar to those found in other year. Consequently, results obtained with this methodology would hold for other years.

It is also important to highlight that as the number of starts of a generator increases, the wear and tear costs are likely to increase as well. These costs will affect the generator's profits and should be considered in future work when a thorough profit study is performed.

\section{CONCLUSION}

This paper defines and quantifies the flexibility in a power system by proposing an index that can be used to measure the technical flexibility of both individual generating units and the overall generation mix. The contribution that a generating unit makes to the flexibility of the generation mix can be gauged by comparing its index to the index of the whole system. The validity of grouping generating units using this index is verified using test cases.

A unit construction and commitment (UCC) algorithm is introduced to determine the optimal investments in additional flexible units required to cope with increasing wind penetration. A case study shows that the technique proposed is able to make the right decision in terms of selecting the right units that should be built to upgrade the flexibility of the whole system to enable it to accommodate more WG.

It is then shown how the economic value of providing flexibility can be assessed by calculating the normalized profits that a generating unit can achieve in the day-ahead and 
balancing markets. Tracking the evolution of this profitability as a function of the wind penetration provides a means to determine when additional investments in flexibility would be desirable.

Finally, the effect of market design on the flexibility requirements, its flexibility and the system operating cost is demonstrated by introducing a rolling market clearing. It is shown that such a market design improves the use of existing technical flexibility and reduces the need for investments in additional flexibility resources such as agile generating units, demand-side response or energy storage. Market design can therefore be seen as a non-technical source of flexibility.

\section{REFERENCES}

[1] J. Kabouris and F. D. Kanellos, "Impacts of Large-Scale Wind Penetration on Designing and Operation of Electric Power Systems," Sustainable Energy, IEEE Transactions on, vol. 1, pp. 107-114, 2010.

[2] B. C. Ummels, M. Gibescu, E. Pelgrum, W. L. Kling, and A. J. Brand, "Impacts of Wind Power on Thermal Generation Unit Commitment and Dispatch," Energy Conversion, IEEE Transactions on, vol. 22, pp. 4451, 2007.

[3] X. Le, P. M. S. Carvalho, L. A. F. M. Ferreira, J. Liu, B. H. Krogh, N. Popli, and M. D. Ilic, "Wind Integration in Power Systems: Operational Challenges and Possible Solutions," Proceedings of the IEEE, vol. 99, pp. 214-232, 2011.

[4] H. Holttinen, "The impact of large scale wind power production on the Nordic electricity system," PhD Thesis, Department of Engineering Physics and Mathematics, Helsinki University of Technology, Espoo, 2004.

[5] J. C. Smith, M. R. Milligan, E. A. DeMeo, and B. Parsons, "Utility Wind Integration and Operating Impact State of the Art," IEEE Transactions on Power Systems, vol. 22, pp. 900-908, 2007.

[6] R. Tanabe, K.Yasuda, R. Yokoyama, and H. Sasaki, "Flexible generation mix under multi objectives and uncertainties," IEEE Transactions on Power Systems, vol. 8, pp. 581-587, 1993.

[7] J. A. F. Moreno, A. M. Garcia, A. G. Marin, E. G. Lazaro, and C. A. Bel, "An integrated tool for assessing the demand profile flexibility," IEEE Transactions on Power Systems, vol. 19, pp. 668-675, 2004.

[8] M. Black and G. Strbac, "Value of Bulk Energy Storage for Managing Wind Power Fluctuations," IEEE Transactions on Energy Conversion, vol. 22, pp. 197-205, 2007.

[9] V. L. F. d. P. d. Silva, "Value of flexibility in systems with large wind penetration," PhD Thesis, Imperial College London, London, 2010.

[10] J. Smith, E. DeMeo, B. Parsons, and M. Milligan, "Wind Power Impacts on Electric-Power-System Operating Costs: Summary and Perspective on Work to Date," presented at the American Wind Energy Conference, March 2004.

[11] B. Parsons, M. Milligan, J. C. Smith, E. DeMeo, B. Oakleaf, K. Wolf, M. Schuerger, R. Zavadil, M. Ahlstrom, and D. Y. Nakafuji, "Grid Impacts of Wind Power Variability: Recent Assessments from a Variety of Utilities in the United States," presented at the the European Wind Energy Conference, Athens, Greece, Feb 27-Mar 2, 2006.

[12] M. Milligan and B. Kirby, "The impact of balancing areas size, obligation sharing, and ramping capability on wind integration," in Proc. 2007 Wind Power Conference \& Exhibition, pp.40.

[13] M. A. Ortega-Vazquez and D. S. Kirschen, "Estimating the spinning reserve requirements in systems with significant wind power generation penetration," IEEE Transactions on Power Systems, vol. 24, pp. 114 124, 2009.

[14] R. Doherty and M. O'Malley, "A new approach to quantify reserve demand in systems with significant installed wind capacity," IEEE Transactions on Power Systems, vol. 20, pp. 587-595, 2005.

[15] F. Bouffard and M. Ortega-Vazquez, "The value of operational flexibility in power systems with significant wind power generation," in IEEE Power and Energy Society General Meeting, 2011.

[16] E. Lannoye, D. Flynn, and M. O'Malley, "The role of power system flexibility in generation planning," in IEEE Power and Energy Society General Meeting, 2011.

[17] N. Menemenlis, M. Huneault, and A. Robitaille, "Thoughts on power system flexibility quantification for the short-term horizon," in Power and Energy Society General Meeting, 2011 IEEE, 2011, pp. 1-8.

[18] A. J. Wood and B. F. Wollenberg, Power Generation, Operation and Control, 2nd ed. New York; Chichester: Wiley, 1996.

[19] R. Baldick, "The generalized unit commitment problem," IEEE Transactions on Power Systems, vol. 10, pp. 465-475, Feb. 1995.

[20] J. Ma, D. S. Kirschen, R. Belhomme, and V. Silva, "Optimizing the flexibility of a portfolio of generating plants," in Proc. 2011 17th Power System Computation Conference (PSCC), Stockholm, Sweden, Aug 22-26, 2011.

[21] D. Kirschen and G. Strbac, Foundamentals of power system economics. Chichester: John Wiley \& Sons, Ltd, 2004.

[22] F. Bouffard and F. D. Galiana, "Stochastic security for operations planning with significant wind power generation," in IEEE Power and Energy Society General Meeting, 2008.

[23] M. A. Ortega-Vazquez and D. S. Kirschen, "Assessing the Impact of Wind Power Generation on Operating Costs," Smart Grid, IEEE Transactions on, vol. 1, pp. 295-301, 2010.

[24] R. B. Johnson, S. O. Shmuel, and J. S. Alva, "Equity and Efficiency of Unit Commitment in Competitive Electricity Markets," the Program on Workable Energy Regulation (POWER), Berkeley, California,1996.

[25] A. Tuohy, E. Denny, and M. O'Malley, "Rolling Unit Commitment for Systems with Significant Installed Wind Capacity," in IEEE Power Tech, Lausanne, Switzerland, 2007.

[26] C. Grigg, P. Wong, P. Albrecht, R. Allan, M. Bhavaraju, R. Billinton, Q. Chen, C. Fong, S. Haddad, S. Kuruganty, W. Li, R. Mukerji, D. Patton, N. Rau, D. Reppen, A. Schneider, M. Shahidehpour, and C. Singh, "The IEEE Reliability Test System-1996. A report prepared by the Reliability Test System Task Force of the Application of Probability Methods Subcommittee," IEEE Transactions on Power Systems, vol. 14, pp. 1010-1020, 1999.

[27] Modeling with Xpress-MP [Online]. Available: http://www.dashoptimization.com/home/downloads/pdf/Modeling_with Xpress-MP.pdf

[28] C. Wang and S. M. Shahidehpour, "Effects of ramp-rate limits on unit commitment and economic dispatch," IEEE Transactions on Power Systems, vol. 8, pp. 1341-1350, 1993.

[29] T. Boehme, J. Taylor, A. R. Wallace, and J. W. Bialek, "Matching renewable electricity generation with demand," Scottish Executive, Feb. 2006.

[30] G. Giebel, P. Sørensen, and H. Holttinen, "Forecast error of aggregated wind power," Risø National Laboratory, April, 2007.

[31] A. Rosso, J. Ma, D. S. Kirschen, and L. F. Ochoa, "Assessing the contribution of demand side management to power system flexibility," in Proc. the 50th IEEE Conference on Decision and Control and European Control Conference, Orlando, FL, USA

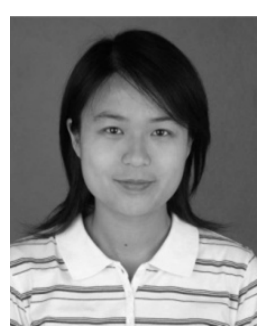

Juan Ma was born in China, 1983. She received her B.Sc. and M.Sc. degree in Electrical Engineering from Tsinghua University, China, in 2006 and 2008. She received the Ph.D. from The University of Manchester, UK, in 2012. Her main research interest is power system operation with wind penetration and electricity market.

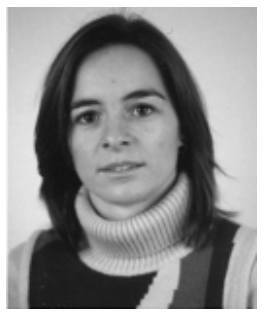

Vera Silva received a B.Sc. and M.Sc. degrees from the University of Porto, Portugal, in 1999 and 2003, respectively, and a Ph.D in Electrical Engineering from the Imperial College London in 2010. She is a Project Manager and Research Engineer at the Department of Economic and Technical Analysis of Energy Systems in the R\&D Division of EDF SA. Before joining EDF she held a position of Assistant Professor at the Polytechnic Institute of Porto (20002005) and a research position at Imperial College London (2005-2009). Her research interests include integration of intermittent generation, demand side management and electric vehicles. She was a member of former CIGRE WG C6.09 on Demand Side Response. 


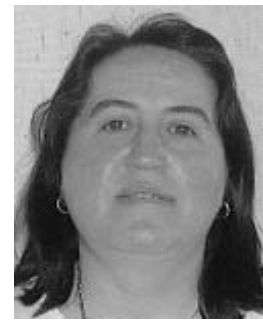

Régine Belhomme (S'83, M'91) received the Electrical Engineering degree in 1986 and the $\mathrm{PhD}$ degree in 1990, both from the University of Liège, Belgium. She is Project Manager and Senior Engineer in the Department of Economic and Technical Analysis of Energy Systems, in the R\&D Division of EDF SA. Before joining EDF, she was with the Research Institute of Hydro-Quebec (IREQ) Canada, where she carried out studies on the integration of Distributed Generation (DG) into the Hydro-Quebec distribution network. She joined EDF R\&D in 1998 and her main activities first concerned the integration of DG and Renewables into transmission and distribution power systems. She is now involved in activities on demand side integration and the development of active demand. She is the Technical Manager of the ADDRESS European Project. She is a member of IEEE, CIGRE and SEE. She was a member of former CIGRE WG C6.09 on "Demand Side Response" and she is a member of CIGRE WG C6.20 on the "Integration of electric vehicles into power systems".

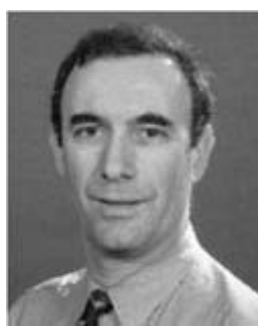

Daniel S. Kirschen (M’86, SM’91, F’07) received the electrical and mechanical engineer's degrees from the Université Libre de Bruxelles, Belgium, in 1979 and the M.S. and Ph.D. degrees from the University of Wisconsin, Madison, in 1980 and 1985, respectively. He is currently Close Professor in Electrical Engineering at the University of Washington.

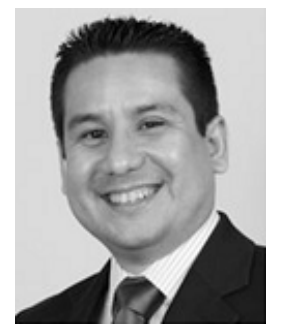

Luis F. Ochoa (S'01, M'07, SM'12) received the B.Eng. degree from UNI, Lima, Peru, in 2000 and the M.Sc. and Ph.D. degrees from UNESP, Ilha Solteira, Brazil, in 2003 and 2006, respectively.

He is a Lecturer in Smart Distribution Networks at The University of Manchester, UK. His current research interests include network integration of distributed energy resources and low-carbon future distribution networks. 